\title{
Rainer Adolphi, Berlin \\ >Personen< ohne Subjekt, Individuen auf Der Suche NACH IHREM AUTHENTISCHEN WER \\ Widersprüche des Person-Seins unter Bedingungen \\ MODERNER TECHNISCHER WELT
}

Marek Siemek zum Gedächtnis

\section{Ein Projekt}

>Moderne ‘ war, was immer man als ihren Beginn ansetzt, stets ein Versprechen: das Versprechen auf Bewältigung bisheriger Lebenseinschränkungen, auf Lösung für die menschliche Bedrängnis und Knechtschaft, Versprechen auf die endlich erreichte humane Welt. Mit den Hoffnungen, mit dem Weg der Moderne in den Zustand zu kommen, der ins Freie führt, stets verbunden waren die Erfahrungen früherer Ohnmächtigkeit - sozial wie im Naturschicksal -, Leiden und Ungerechtigkeit. Bzw. die Ängste, dorthin zurückzufallen.

Und zugleich ist der reale Eintritt in diese Moderne - wie kein anderes Zeitalter - eine Epoche des eigenen Zwiespalts, in sich zerfallen. Seit mehr als zwei Jahrhunderten gehört »Krise« zur Kernsemantik ihrer Selbstverständigungen, »Krise« sowohl im negativen Sinne eines drohenden Stockens oder Zusammenbruchs wie auch im medizinisch-therapeutischen Sinne der Krisis - der Entscheidung, ggf. des Letzten Gefechts. In Sprachen von »Krise« hat sich, anfangs in erkennbaren Konjunkturen, inzwischen oft nahezu als kontinuierlich mitdrängender Strom, ein Diskurs über den Prozess der >Moderne < etabliert, über Wesen, welthistorische Bedeutung und zu gewärtigende Perspektive (Zukunft). >Moderne ist so in der doppelten Hinsicht ein Projekt: eine reale Umgestaltung von Wirklichkeiten, Handlungs- wie Denkmöglichkeiten, menschlichen Mentalitäten und zugleich das Bemühen, zu einer Verständigung über das eigne Zeitalter zu kommen, sowie Auseinandersetzung über die Erfahrungen der Veränderung.

Wesentliche Gestalt dieses Diskurses ist die Kritik. Die sich installierende Moderne: ihr Prozess ist entsprechend seit mehr als zwei Jahrhunderten begleitet von einer ihn in Öffentlichkeit kommentierenden Kritik. Krisenbewusstsein und Diskurs der Kritik, den Prozess selbstbewusst zu machen und die Eindeutigkeit zu forcieren, gehören in ihr untrennbar zusammen. Es ist die Kritik an Gesamtepoche, an Geburtsfehlern, an >Ideologie` und an einseitigen Entwicklungen, dominant gewordenen Einseitigkeiten in ihr.

Diese Kritik zeigt ihrerseits eine komplexe Lage. Auf der einen Seite der Kampf eines kulturellen und gesellschaftlichen Anti-Modernismus - das Festhalten-Wollen des Alten (〉Guten Alten $<$ ) oder überhaupt das Zurück, die restitutio in integrum. Symbol für >Moderne ist hier seit je der Einschnitt Französische Revolution, daneben die Wendung gegen die Autorität der Kirche; und die Kritik macht sich fest am Verfall von Werten und Ordnung, von Moral und anerkannten Positionen. Typologisch eine zweite Front der Kritik gilt den geistigen Aufwieglern, geistigen Brandstiftern der >Moderne ২: dem zunehmenden Subjektivismus in den Theorien der Philosophie, dann in der Kunst, und bald auch im allgemeinen Denken der Menschen. Symbol für diesen Prozess der zunehmenden Relativierung des Objektiven und der Wahrheit wurde zentral >Nietzsche $<$ - das >Vorsicht Nietzsche!^. Schließlich eine dritte Front: dass die herrschend gewordene Moderne eine halbierte - oder ambivalente - sei. Mit universellen Prinzipien, wie >Vernunft!८, >Freiheit!८, >Recht! u. a. m., gegen das Alte sich aufrichtend, sei es de facto nur die Moderne der imperial gewordenen Systemkriterien eines Teilbereichs - des Szientismus, oder der Ökonomie zum Beispiel; oder de facto nur die Mo- 
derne einer bestimmten sozialen Schicht (bzw. Gruppe) und ihrer Ideale und Interessen - nur die Moderne des Bürgertums, der Gebildeten, des `Mannes`, der westlichen Welt ...

J. Habermas, in seiner berühmt gewordenen Deutung Der philosophische Diskurs der Moderne, hat die Motive dieser Strömungen zusammengebracht. In der so verschiedenartigen Kritik hat er, so lässt sich die Bedeutung seiner Interpretationen verallgemeinern, die geistigen Motivationen, die auf eine Erfahrung der Wirklichkeit reagieren, zu sehen gegeben. Der restaurative \Konservatismus`der ersten Front der Kritik zeigt sich dabei als - neben Statusideologie der Mächte der alten Ordnung und entsprechender >ideologischer Hirtenbriefpolitik - die Projektionen der Modernisierungsängste und Modernisierungsverluste. Denn in der Tat geht mit dem Prozess eine neue Unbehaustheit einher, und setzt der Prozess neue Anforderungen an die Menschen, Anforderungen auf Dezentrierung von Lebenswelten und Subjektverständnissen. ${ }^{1}$ Die Kinder des Aufbruchs demgegenüber, den >Moderne darstellt, hat Habermas' Deutung als gewissermaßen zwei Geschwister bewusst gemacht: als die klassische Vernunft-Orientierung und als die >Romantikı, als die Suche nach einem Menschen-Allgemeinen - darunter vor allem >Wissenschaft sowie universellen rationalen Normen - und als das Pochen auf meine Individualität, meine eigene Stimme, meinen Eigen-Sinn. Die Verwerfungen der Kritik begönnen vielmehr damit, dass mit dem Denken, wofür Nietzsche steht, jene zweite Seite, die >Romantikı, zu einer anderen, einer >ästhetischen`Moderne entgrenzt worden sei - welches dann wiederum am Ende des 20. Jahrhunderts die >Postmoderne in universelle Theorie gegossen habe. ${ }^{2}$

Moderne zeigt sich so wesentlich als der Kampf mit sich selbst. Wo nicht nur Flucht aus dem Zeitalter, seinen Wirklichkeiten und Forderungen, ist die Kritik, ihr selbst oft unbewusst, zugleich Teil der Arbeit an der Moderne: ist - in vielen ihrer stärksten Ausprägungen geradezu daraus erwachsend -, aus >romantischem` Geist Errungenschaften und neue Möglichkeiten der Epoche einzuklagen gegen Einseitigkeiten und Folgen der herrschenden Gestalt des Lebens. Der Streit geht um die Deutungshoheit über das Zeitalter.

Das Kritische, weil aus Erfahrungen erwachsend, ins Recht setzen zu müssen - Recht subjektiv und auch betreffend relativ-objektiv: als das darin sich Aussprechende -, verschärft die Maßstäbe für den Theoriestatus von Aussagen über die Moderne. Vor allem gilt die Frage dem Verhältnis von deskriptivem Gehalt und normativen Grundlagen, Normativität eines Ansatzes wie Normativität der Kritik. - Das Deskriptive wäre in Konsequenz die gesellschaftswissenschaftliche Analyse: aus einem Sensorium für die Ausprägungen und lebensweltlichen Problematiken, aber auch für Nebenfolgen und historische Kontingenzen, >Moderneく als einen schwierigen, plural verfassten (multifaktoriellen) und stets singulären Transformationsprozess vor die Reflexion zu bringen. In dieser Hinsicht ist, was die heutigen Diskussionen aus den Auseinandersetzungen über das > Wesen $<$ der Epoche und aus der so vielgestaltig die Erfahrungen begleitenden Kritik gezogen haben, wohl an erster Stelle das, von verschiedenen >Modernen zu sprechen - von gesellschaftlicher, technischer, wissenschaftlicher, ökonomischer, kultureller, ästhetischer Moderne, von einer Moderne des >Lebensstils`, und auch dem Diskurs über die neuen Wirklichkeiten. Als deskriptiv fruchtbar, fruchtbar für das analytische Potential der konkreten Thematisierung, gilt es inzwischen, von solch verschiedenen >Modernen` und von entsprechenden spezifischen Prozessen der Modernisierung auszugehen; verschieden auch, was ihre zeitlichen Verläufe - und typischen Epochen - betrifft. ${ }^{3}$ Zudem

1 Das gibt der Rede von der `Bewährtheit` des Alten, gegenüber den (befürchtet) waghalsigen Experimenten und (verblendeten) Kopfgeburten des Neuen, ihr subjektives Recht.

2 J. Habermas, Der philosophische Diskurs der Moderne, Frankfurt/M. 1985.

3 Diese Weite des Blicks scheint andrerseits häufig auch ihren Preis zu haben: einen nicht unerheblichen epistemischen Preis. Deskriptive Distinktion scheint ab einer gewissen Schwelle nicht zu haben zu sein, ohne dass die Rede von >Moderne $<$ - etwa `technischer Moderne $<$ - zu reinen Beschreibungstiteln für ein abgestecktes Feld wird; Beschreibungstitel, die schon nicht einmal mehr eine theoretische (das heißt: mehr denn untersuchungspragmatische) Konzeption über die jeweilige betreffende Gegenstandssache 
gibt es offenkundig verschiedene Wege zur Moderne, wie uns heute an der islamischen Welt oder an China und anderen Schwellenländern massiv ins Bewusstsein tritt.

Ich möchte dies im Folgenden mit einer These, die das Selbstbild der Subjekte in diesem $\mathrm{Zu}-$ stand >Moderne < betrifft, erweitern; und ich möchte dazu eine Problemskizze im Anschluss an eine Einsicht Hegels entwickeln. Die Rede von den verschiedenen >Modernen lationell-vielen Wegen zur Moderne hat auch etwas Irisierendes. Wo sie zum Selbstverständlichen des Diskurses und der Wissenschaft zu werden beginnt, ist mit der Evidenz des Materials zugleich oft auch alle Begründung der Kriterien für >Moderne $<$ - sowie Kriterien der Bezeichnung zu kritisierender Entwicklungen - dem Geschichtlich-Faktischen überantwortet. Anhand von Hegels betreffender Argumentation dagegen lässt sich zeigen, wie deskriptive Perspektiven und Begrifflichkeiten sich der Vielgestaltigkeit und auch empirischen Kontingenz des Geschichtlichen analytisch anschmiegen können und doch in eine theoretische Reflexion und Begründung normativ-kritischer Positionierung eingebunden bleiben. - Dazu sei die vielleicht einzige Stelle, bei der bei Hegel eine gesehene >Dialektikı des Geschehens keine >höhere` Vermittlung und Versöhnung hat, verallgemeinert, in einer verallgemeinerten Weise verstanden: die bekannte Stelle Rechtsphilosophie § 245, dass die Moderne als gesellschaftliche Wirklichkeit, d.i. »Die bürgerliche Gesellschaft«, bei all »dem Übermaße des [von ihr geschaffenen und entbundenen] Reichtums [...] nicht reich genug ist, d.h. an dem ihr eigentümlichen Vermögen nicht genug besitzt, dem [gleichzeitig] Übermaße der Armut und der Erzeugung des Pöbels zu steuern «. ${ }^{4}$

Diese Hegel'sche Einsicht möchte ich, die heutigen Wirklichkeiten zwei Jahrhunderte nach den Erfahrungen Hegels vor Augen, dergestalt weiterdenken, dass das »nicht reich genug« für so gut wie alle Dimensionen des Prozesses der Moderne gilt, keineswegs einzig für das handfest MateriellÖkonomische. ${ }^{5}$ Moderne ist, bei all ihrer Entbindung von ökonomischer, geistiger, wissenschaftlicher Produktivität - auch allseitigem nüchternem Wissen sowie moralischer und psychologischer Reflexion -, eine Umwandlung der Lebensrealitäten, bei denen diese Potenzen dennoch nicht gro $\beta$ genug sind, um der Kehrseite ihrer eigenen neuen Wirklichkeit gewachsen zu sein - und die Innenwelten der Subjekte, das mentale Profil ihrer Selbstverständnisse, sind die Stelle, an der sich dies in einer anonym-unbewussten, indes lähmend-zerstörerischen Weise austrägt.

\section{Die Mächte der Modernisierung (Die Wirklichkeit eines Zustands)}

\section{Auch ein profanes Geschehen}

\section{(Die Notwendigkeiten des sozialwissenschaftlichen Blicks)}

Die Macht der Moderne, d.h. wodurch sie sich durchgesetzt hat und in neuen Weltregionen immer weiter sich durchsetzt, ist in Teilen auch eine von durchaus profanen Konstellationsfaktoren. Dies entschieden mit im Blick zu haben bringt eine Eingrenzung und Zuspitzung des Problems der Moderne und Problems auch ihres Kampfes mit sich selbst.

beinhalten - also etwa eine Konzeption über das Technische und seinen (wechselseitigen) Zusammenhang mit z. B. dem Kulturellen.

4 Vgl. Grundlinien der Philosophie des Rechts, §§ $241 \mathrm{ff}$.

5 Schon für Hegel waren »Armut« und »Pöbel« nicht - nicht nur - eine Wirklichkeit der ökonomischen Lebenslage, sondern, noch vor den dann daraus folgenden äußeren Ausprägungen der Alltagsexistenz dieser Schichten, ein »sittliches « Problem der Gesellschaft als ganzer: diese Existenz deswegen den Menschen korrumpierend, weil er sich darin als ent-wertet erfährt, als zur bloßen Masse gepfercht, als vorenthaltene Würde des Lebens. 
Das trifft in besonderem Maße zu auf die für die konkrete Analyse zu unterscheidenden >Modernen des Ökonomischen, Technischen, Ästhetischen usw. und die verschiedenen Wege zur Moderne. Die unterschiedlichen >Modernen Prozesse, zur gesellschaftlich bzw. sozial dominierenden Wirklichkeit zu werden, eine Reihe ganz differenter Geschehens- und Ursachenformen. Manche kommen - etablieren sich - hauptsächlich via Selektion: dass Anderes, Vormaliges (oder zeitgleich Alternatives) sich nicht halten kann dagegen; manche via Einsickerung (von den Rändern bzw. außen her) und langsame Gestaltveränderung des Alten dadurch; manche durch bewussten Diskurs, und Reflexionsanstrengung der Optimierung resp. Suche-nach-Lösungen; ${ }^{6}$ manche durch de facto Umsturz, bes. Umsturz in den herrschenden Eliten (Träger-Gruppen des Jeweiligen); manches auch schlicht mangels Alternative - für den betreffenden Sachverhalt ohnehin der einzige herausgebildete (oder vor Ort bewusste) >Kandidat gewesen zu sein; und manches schließlich wohl auch geradezu anonym, ohne ein erkennbares Durchwas? oder Von-wo-aus? - ein irgend sich ergeben habender Keim der Innovation und scheinbar kontingente, kaum sinnhaft nachzeichenbare Ausstrahlungen bzw. Übernahmen.

Diese Prozesszusammenhänge, die im Einzelnen bestehen, verschärfen die Frage nach dem Allgemeinen >der Moderne: die Anforderungen an eine Bestimmung. Sich die möglicherweise mitspielenden ganz differenten Geschehens- und Ursachenformen bewusst zu machen, lässt zunächst vor allem einiges deutlicher sehen, was bei allzu hoher - allzu >philosophischer $<$ - Rede von >Moderne tendenziell nicht im Blick ist. Genannt seien da nur vier zentrale Punkte. ${ }^{7}$

Das Erste haben heutige Theorien bereits weithin verinnerlicht: Dass >Moderne $<$ nicht einfach eine Stufe der Verbesserung - ein Fortschritt - auf einer generellen Skala menschlicher Zivilisation ist, sondern, und das gilt selbst für die Modernisierung im Ökonomischen und im Bereich des >Technischen`, die Ablösung oder Verdrängung der Strukturprinzipien eines Alten. >Moderneく definiert auch die Prinzipien um, nach denen ein Zustandsgefüge - und etwas in einem Zustand - als rational, funktional, angepasst usw. sich ausweist bzw. gilt. ${ }^{8}$ Ohne dass dies gleich einer totalen Relativierung Tür und Tor öffnen würde, gilt es in Rechnung zu stellen, dass es Prinzipien gab, nach denen auch das Alte oder Nicht-Moderne - solange es herrschte - relativ zu seiner Zustandswirklichkeit rational, funktional, angepasst usw. war, d.h. ohne schlechterdings ein Drängen-zur-Moderne.

Dann vor allem zweitens: Dass jeder Prozess der Zivilisation in vielerlei Hinsicht nicht ein Prozess-ins-Offene, causa sui einer Schöpfung ist, sondern materialiter Naturgegebenheiten umwandelt und Ressourcen, die in ihn eingehen, vernutzt - allgemein, Ressourcen aus der psychischen und ökologischen >Umwelt< des Systems. Wegen dieser materiellen Seite hat der Prozess auch Grenzen: Grenzen oder einen >Grenznutzen gleichem Maße, wie es in ihn eingeht, wiederum neu generiert, nachbrütet. Kein Zustand ist ein geschlossenes System, und das gilt für die jederlei Moderne kennzeichnende Dynamisierung sowie sich steigernde Beschleunigung aller Geschehnisse in besonderem Ausmaß. Das ist überall dort

6 Einiges sogar wohl auch als: entstandene (oder aufgebrochene) Problemerfahrung und herausgebildete/ entwickelte `Technik zur Bewältigung - als Ketten von challenge-and-response.

7 Hinzu kommt das ohnehin Evidente von schlicht Ungleichzeitigkeiten in der Entwicklung, die durch ihr Nebeneinander bzw. ihre Überkreuzung ihrerseits zu Spannungen führen und zuweilen auch Widersprüche der Dynamik als noch zerreißender erfahren lassen. Zum Beispiel zwischen ökonomischer und gesellschaftlich-politischer Modernisierung; oder zwischen technischer/wissenschaftlicher und Modernisierung der sozialen Werte bzw. Lebensziele/Rollenidentitäten.

8 Die neuere Wissenschaftstheorie und Blick auf die Wissenschaftsgeschichte kennt dies inzwischen in vielfacher Weise. Aber auch schon Max Weber hatte dies als den fruchtbaren Ansatz für die (vom >Protestantismusく ausgelöste) Heraufkunft der `moralischen` Moderne erkannt (vgl. Die protestantische Ethik und der Geist des Kapitalismus, 1904/05). 
ausgeblendet, wo >Moderne $<$ - der Eintritt in die Epoche $>$ Moderne $\prec$ - als rein geistiger Sachverhalt gesehen ist: >Moderneく als geistige Epoche, geistige Einstellung, geistige Option.

Bei hoher Rede von >Moderne< tendenziell nicht im Blick ist drittens: Dass ein Schub der Moderne stets dergestalt neue Kriterien bedeutet - Kriterien auch des \Erfolgs neuen Wirklichkeit -, dass nicht, oder jedenfalls nicht unmittelbar, alle in gleicher Weise daran teilhaben. ${ }^{9}$ Kein Schub oder Schritt - und die mit ihm herrschend gewordenen Kriterien - bedeutet eine neutral-gleiche Veränderung für die Lebensbedingungen in allen Bereichen und aller davon Betroffenen. Jeder Schub vielmehr ist mit gewisser neuer Selektion verbunden: Selektion von relativ besser- und relativ schlechtergestellten Menschen-Gruppen und auch subjektiv von gruppenspezifischen Lebenswelten, die den Veränderungen und deren Phänomenen/Ausprägungen/Folgen eher affin sind oder sie eher als hereinbrechendes Fremdes, als Bedrängung und Beängstigung erleben lassen.

Schließlich viertens: Dass ab einer bestimmten Schwelle, Schwelle bes. der Dynamisierung in sich, die mentalen Kapazitäten bzw. Bedürfnisse - die von Einzelnen und die von Gemeinschaften zunehmend überfordert sind, weil der Prozess der Moderne die Funktion, die das Alte hier hatte, nicht vollständig ersetzen - das Alte raufheben $<$ - kann. Das macht das allgemeine Unbehaust und Unbehagen in der Moderne.

\section{»Nicht reich genug«}

Anforderungen mithin an eine konkretionsangemessene Bestimmung von >Moderne ২. Die Wirklichkeitsgegebenheiten markieren einen Stand der Komplexität, unterhalb dessen eine Theorie ein zu ungebrochenes Bild zeichnen würde. - Dass die Gesellschaft, auf ihrem Weg der Moderne, »nicht reich genug« ist, um Ausprägungen und Folgeerscheinungen ihres Prozesses integral eingebunden zu halten, bedeutet darum dann, über die von Hegel hier direkt angesprochenen durch schlicht die ökonomischen Subsistenzbedingungen gegebenen Verwirklichungsmöglichkeiten des Lebens hinaus, ebenfalls ein Mehrfaches.

- Um dies in Stichworten zu umreißen, so erstens »nicht reich genug«, denn es gibt unleugbar Modernisierungs-Verlierer - Gruppen und Generationen, gesellschaftliche Bereiche und auch Landschaften (oder Weltgegenden), die in der betreffend modernisierten Wirklichkeit relativ zurückfallen; oder ganz herausfallen. ${ }^{10}$ Verlierer in diesem Prozess gibt es nicht nur in ökonomischer (genauer: >wohlstands $<$-relativer) Hinsicht, sondern auch in jenen, bei denen die Grundlagen, worauf ihr bisheriger Status und Prestige basierte, sich sukzessive auflösen - und die Gesellschaft als Ganzes »nicht reich genug« ist, um diese Ent-Wertung durch neue Nischen, neue Identitäts-Angebote oder schlicht soziale Transferleistungen aufzufangen. Anders gesagt, es gibt in diesem Prozess beständig - und im Großen gesehen: zunehmend - Zonen, wo es nicht um irgendwelche >Gerechtigkeit oder ähnliches geht, sondern nur noch um soziale Beschwichtigung und Befriedung; nur noch darum, etwas nicht überkochen zu lassen.

- Dann, ebenfalls »nicht reich genug«, insofern die Teilhabe an den neuen Wirklichkeiten zunehmend in einem viel größeren Maße hohe Bildung und Wissen erfordert, die nicht in allen Bereichen der Gesellschaft in zulänglicher Qualifikation bei den Menschen herangeformt werden können. Selbst einfachere Tätigkeiten im Handwerk, oder das frühere soziale Auffangbecken >Polizeiく und >Militär`, erfordern heute meist hohe technische, mathematische und naturwissenschaftliche Qualifikationen. Anteilsmäßig wird, was seit den anfänglichen Phasen des Eintritts in die industrielle Moderne zunehmend überwunden schien, die Gruppe derer, die mangels Qualifikation - Standard-

9 Das gilt selbst für das Vorzeige-Projekt der Moderne: die Modernisierung von Rechtssystemen.

10 Dies wohl auch schon immer in diesem Prozess der Moderne, nicht erst heute, sondern strukturell nicht anders in früheren Phasen. 
qualifikation - nur noch für Sub-Tätigkeiten am unteren Ende der sozialen Leiter taugen, wieder immer größer. ${ }^{11}$

- »Nicht reich genug« drittens, weil ab einer bestimmten, heute jedenfalls erreichten Schwelle die Dynamik der Modernisierung und Modernisierungsanpassungen - schon allein auf die objektive Zivilisationswelt in sich gesehen - zu schnell geworden ist. Beherrschte Praktiken und Techniken, aber auch produzierte Güter kommen zu schnell außer Dienst: eine immer größere Verschleuderung von Kapazitäten, weil etwas - Menschen wie Waren -, das eigentlich noch >funktionsfähig ‘ ist, im aktuell herrschenden Neuesten nicht mehr funktional oder überhaupt verwendungsfähig ist.

- »Nicht reich genug «, als dass der Prozess kulturelle Orientierungen - kulturelle Selbstverständlichkeiten - in gleicher Weise, wie der Wandel Bisheriges zum Veralteten werden lässt, nachwachsen ließe: in gleicher Weise die Sicherheit eines Vertraut-Gültigen, etwas, das nicht jeweils neu ausgehandelt werden muss oder auf Risiko der persönlichen existenziellen Urteilsüberzeugung übernommen werden muss. Die Decke habitueller kultureller Selbstverständnisse und Orientierungen wird dünner.

- Fünftens »nicht reich genug«, weil die internen Steigerungszwänge so groß sind - das System inzwischen so dicht, verurteilt zur Potenzierung -, dass, gefesselt im Netz der Imperative des $>$ Noch mehr Modernität!^(oder doch >Mindestens so viel Modernität wie die herrschenden Wirklichkeiten!〈), Entscheidungs- und Handlungsspielräume, Verantwortlichkeiten und das Wer-eigentlich? zunehmend nicht mehr sichtbar sind. Und auch die Einzelnen in ihrem Wägen und Tun orientieren sich viel stärker wieder an Parametern situationslokaler Konstellationen wie Akteursrollen - einem Umgrenzt-Lokalen, das immer weniger durchgeschlossen ist mit übergreifenden Wert-Prinzipien. Zwischen lokalen Horizonten (sowie entsprechend fragmentarisierten Identitäten), in denen für die in sie verwobenen Einzelnen ein >moralischer Sachverhalt bzw. eine >moralische< Tragweite in der Tat häufig nicht mehr unmittelbar manifest ist, und andererseits den großen hohen Wert-Allgemeinheiten breitet sich ein Wert-Vakuum aus, subjektiv wie objektiv.

- Schließlich, dass die inneren Aufgliederungen und Differenzierungen der gesellschaftlichen Bereiche - in der Modellierung der klassischen soziologischen Theoriebildung: die >Arbeitsteilung < - so komplex geworden sind, dass die immer modernere Gesellschaftswirklichkeit auch bezüglich der gesellschaftlichen Solidarität - der Solidaritäts-Horizonte - »nicht reich genug « ist: trotz aller Erfolge und Lebensentlastung und trotz aller moralischen Diskurse nicht reich genug an der Generierung innergesellschaftlicher (gar internationaler) Solidarität. -

Dies diagnostisch das Unreine, das >Moderne, sobald konkret als ihre Prozesse der Modernisierung gesehen, zeigt. Es bedeutet ein »nicht reich genug«: auf der Kehrseite der einhergehenden Ent-Wertungen; im Hinblick auf Bildung, dem Ausmaß der bereitstellbaren Anleitungen dazu; beim Einsatz von humanen und ökonomischen Qualitäten, mit deren Schätzen exzessiv und kurzfristig-denkend umgegangen wird, nicht haushälterisch oder gar nachhaltig; bei dichten kulturellen Selbstverständlichkeiten, die auf der Höhe der Wirklichkeiten sind, nicht in ihrer Habitualisierung obstruiert werden oder doch hinterherhinken - anders gesagt, ein »nicht reich genug« an Wert-Erfahrungen; ebenso ein »nicht reich genug« an Akteurs-Verständnissen, die mit den neuen Erfahrungswelt-Konstellationen und Mechanismen der Wirklichkeit Schritt halten, dem Agierend-Geschehenden seine Verstehbarkeit ermöglichen, sein humanes Antlitz; und schließlich an moralischer Solidarität, Horizonten des Solidar-〉Wir ${ }^{12}{ }^{2}$ Sie alle haben sinnfällig strukturelle Ursachen, die mit

11 Und, nebenbei: Die Menge der neuen Super-Models und neuen Super-Popstars, die die Gesellschaft braucht, hält sich - entgegen dem, was entsprechende wuchernde Casting-Shows suggerieren - doch sehr in Grenzen ... Diese um sich greifenden Träume, vorbei an jedem Weg der Bildung und des Wissens zu einer (hohen) Position in der Gesellschaft zu kommen, sind vielmehr Index für das erfahrene Nicht-mithalten-Können mit den Anforderungen selbst an einfachere Tätigkeiten in der sozialen Hierarchie.

12 Bis zur Zersplitterung der Generationen, dem Brüchigwerden des Zusammengehörigkeitsempfindens, einer >natürlichen` Generationen-Gerechtigkeit. 
diesem Zeitalter und den Weisen seiner Etablierung zusammenhängen. ${ }^{13}$ Die Wirklichkeit der Moderne produziert Felder von realen Erfahrungen, die dann in der Moderne-Kritik sich aussprechen; die den Prozess der Moderne begleitende Gegen-Rede hat ihre Quellen in diesem selbst, und das, neben dem von Hegel hier direkt angesprochenen Problem des gerade modernen Pauperismus, in einer mehrfachen, hoch komplexen Gestalt.

Für eine entsprechend grundlegende Perspektive auf die Verschlungenheit von Moderne und Moderne-Kritik, die mit sozialwissenschaftlichen Betrachtungen, wie sie hier evidentermaßen mit erforderlich sind, konstruktiv ineinanderzugreifen vermag, dabei besonders signifikant ist weniger die thematisierende große Kritik, die die erfahrenen Negativausprägungen in gerader Rede ausspricht, sondern sind die sozusagen inversen und verschiebenden Formen des Nicht-in-der-Moderne-Heimischseins ihrerseits - plakativ gesagt, die >falsche〈 Kritik, Ersatz- und Sündenbock-Kritik. Das sind, und dies in wohl in allen Phasen der Moderne, die hauptsächlich sozialpsychologischen Pathologien, dass die erfahrenen strukturellen Wirklichkeitsdefekte personalisiert werden und bestimmte Akteurs-Gruppen zum Ziel projizierter Bedrängnis oder vollends Verschwörung werden. In den Bruchstellen und Lücken des nicht lebensweltlich-zureichend in den Prozess Integrierten (oder mit dem Prozess Mithaltenden) kommt es zum Aufkeimen von Ressentiments: Ressentiments vor allem, um einem Unverstehbaren einen handelnden Verursacher zu schaffen. Das sind nationalistische Ressentiments wie auch solche wider >Den Staat $<$ Ressentiments gegen eine gemutmaßte Macht-Clique wie auch die Mutmaßung von Fremdsteuerung, Infiltration, inszenierter Wirklichkeitsinformation - Induzierung von Bildern und Meinungen in den Köpfen - und Verschleierung >Der Wahrheit`; schließlich neue ethnische und religiöse Ressentiments wie andrerseits ein neuer Dünkel gegenüber den `zu wenig Intelligenten`, gegenüber nicht genügend `Leistungsbereiten`, gegenüber >Sozialparasiten< usw.

Darüber hinaus jedoch, dahin möchte ich meine Leitthese präzisieren, emergieren heute gerade auch in den Kernbereichen der Gesellschaft Selbstverständnisse der Menschen, die zunehmend etwas Ir-reales, De-Soziales und Krisenhaftes an sich haben. Etwas Soziales, auch Sozial-Generiertes - von den Einzelnen in ihrem Agieren und Sprechen Vollzogenes - erscheint als naturale Gesetzlichkeiten und Imperative der Welt, alle Diskonformitäten nur als subjektive Fehlanpassungen; Beschreibungen, auch Selbstbeschreibungen, ja deren ganze Sprache und Begrifflichkeiten sind mehr und mehr an den Funktionalitäten der geschaffenen rationalen Gegenstände der Lebenswelt (sowie Mechanismen erzeugten >Wissens $\triangleleft$ ) abgelesen, d. h. an den Funktionalitäten der herrschenden Zivilisation. Und gleichzeitig läuft das Subjektive zunehmend leer, erzeugt Phantasmen der Selbsterfindung, Phantasmen artistischer Verwirklichung und Identität. Das macht die Aufgabe für eine philosophische Theorie.

\section{Szenen der Zivilisation}

\section{(Das Passivum der menschlichen Selbstverständnisse)}

Von Selbstverständnissen zu reden, individuellen, gesellschaftlichen, kulturellen Selbstverständnissen, gehört heute allgegenwärtig zum Diskurs in Wissenschaft und Alltag. Der Begriff legt dabei eine Eindeutigkeit des Sachverhalts und Einfachheit nahe; wie im Allgemeinen benutzt, würde es ihn in die Folge des klassischen >philosophischen Subjektivitäts-Begriffs stellen: eben subjektgesetzte leitende Bestimmungen einer Subjekt-Instanz oder -kompetenz von sich, eine reflexive Subjekt-Bestimmung in Analogie zum Konzept des `Selbstbewusstseins`. Oft ist denn, ohne dass

13 Und: sie sind grundlegend genug, um jeden Gestus der Abwimmelung, die beklagten Seiten der Moderne existierten nur, wie sie >nicht richtig verwirklicht« sei, zu verbieten. 
diese Vagheit offen bewusst wäre, damit nur überhaupt eine - mitspielende, mit zu berücksichtigende - Sachdimension angezeigt. Selbst-Verständnisse jedoch sind sicherlich mehr. ${ }^{14}$

Selbst-Verständnisse, zureichend komplex gefasst, umfassen: Verständnisse des Handelns und des Erleidens; Verständnisse von ımenschliche Akteureく, und was transmenschliche Akteure - Geister, Götter, systemische (oder ggf. institutionelle) Mechanismen, ... - wären und was >Geschick ; Verständnisse von Bedingtheit; und auch Verständnisse des Was-wissbar und Was-unerkenntlich - damit verbunden Verständnisse einer Dynamik (und zumeist Normativität), wo >besseres oder >eigentlicheres` Ich-Sein, Ich-Verwirklichung läge und wie zu erreichen. Solche Selbst-Verständnisse machen konkret Selbst-Bilder: als was den (individuellen, gesellschaftlichen, kulturellen) Subjekten ihr gelebtes Sein und Tun im Großen der Welt vorkommt, und wie sie es sich vorstellen und auch empfinden. Selbst-Verständnisse, Selbst-Bilder der Subjekte werden darin stets bestimmt durch einerseits tradierte kulturelle Muster - also das, was ein klassisches Handlungsverständnis und Verständnis von >Weltbild «, >Paradigma Orientierungen, Aktions-Typen und Wertungen, mit denen die Akteure in ihre Handlungspläne, Überzeugungs-Bildungen und sozialen Interaktionen hineingehen. Selbst-Verständnisse, Selbst-Bilder werden aber auch und wesentlich bestimmt - gleichsam rückwärtig - durch erfahrene Wirklichkeiten.

In allen bisherigen Epochen waren dies: Wirklichkeiten der Handlungs-Horizonte und Handlungs-Möglichkeiten - Kausal-Bewusstsein, Interventions-Tiefe, Stand der Techniken im weitesten Sinne -, Wirklichkeiten der Handlungs-Umwelt - Stoffe, Räume, Schicksale - und drittens soziale Lebenswirklichkeiten, genauer gesagt soziale Lebenswirklichkeiten, die ich als solche erster Stufe bezeichnen möchte - das meint, klassischerweise festgelegt durch gesellschaftliche Stratifikation, Zugang zu Gütern usw. In allen bisherigen Epochen haben die erfahrenen Wirklichkeiten sich so nur vor allem in Handlungs-Anpassungen eingeprägt, entsprechenden Anpassungen auf Seiten der getätigten kursierenden Handlungsakte und Praxen. ${ }^{15}$ Im heutigen Stadium der Moderne jedoch sind dies, strukturell eine neue Epoche, zunehmend Wirklichkeiten, die durch ihrerseits geschaffene Rationalitätsverkörperungen gebildet sind, Wirklichkeiten, die von zu intellektuellen oder materialen $\mathrm{Ge}$ genständen gewordenen geistigen Ideen sozusagen bevölkert sind. Das Agieren - im Theoretischen wie Praktischen - bewegt sich zunehmend in einer Welt von Beständen des >Wissens 〈 und vor allem von technischen Apparaturen. Die letzte Stufe, in manchen Bereichen bereits erreicht, ist, wenn die Omnipräsenz zu wissenden >Wissens , die verkörperten Rationalitätsobjekte, die Apparate - wenn diese ihrerseits Wirklichkeiten generieren, die wie `natürliche` Wirklichkeiten aussehen; oder doch durch ihre schiere Massivität den Unterschied entschwinden machen - gar nicht mehr empfinden lassen, was die primäre naturgegebene und zwischen-menschliche Lage des Handelns, Denkens und Lebens, die conditio humana einmal war.

14 Von der logischen Problematik - und >philosophisch-idealistischen ( Versuchung von Konzepten von der Struktur von >Selbstbewusstsein $<$ - noch ganz abgesehen. Vgl. dazu etwa K. Cramer (Hg.), Theorie der Subjektivität, Frankfurt/M. 1987; E. Tugendhat, Selbstbewusstsein und Selbstbestimmung, Frankfurt/M. 1979.

15 Also dass das Subjekt-Profil der Selbst-Verständnisse und Selbst-Bilder doch im Wesentlichen mit den betreffenden - gleichsam höheren - kulturellen Mustern übereinstimmt (das heißt: übereinstimmend bleibt), lediglich ergänzt oder angereichert um Paradigmen der Umsetzung im Konkreten, die sich um des Verwirklichungs- (und Bestätigungs-) Erfolgs willen herausgeformt haben - kondensierte Anpassungen entsprechend Gegebenheiten. 


\section{Bilder-Verhalten}

Was dies an Einschnitt und Umbesetzungen bedeutet, die die bisherigen theoretischen Konzepte herausfordern, hat, neben den bekannten >postmodernen Theorien der >Simulation`, der >Beschleunigung «, der neuen Feier der `Simulacren unserer Welt - also: Baudrillard, Deleuze, Virilio u. dgl. ${ }^{16}$ etwa Vilém Flusser zu begreifen unternommen. In den 1980er Jahren, als die heutige Zuspitzungen durch Automatisierungstechnologien, selbstlernende Computerprogramme, Internet und Virtuelle Welten allenfalls in ihren Anfängen standen, man die Veränderungen durch Datennetze, bildgebende Verfahren, elektronische Bildbearbeitung und -manipulation, >intelligente Unterstützungen` (wie z. B. im Auto), elektronische Finanzmärkte, Computerspiele und elektronische Animationen ${ }^{17}$ usw. noch kaum kennen konnte, hat er das unaufhaltsame Entstehen eines neuen Zeitalters, einer gänzlich neuen Struktur von Gesellschaft und Leben kommen sehen. ${ }^{18}$ Diese ist gekennzeichnet durch die Entwicklung der Technik zum Apparat - Durchsetzung der Wirklichkeit, mit der umzugehen ist, mit Apparaten, genauer Apparatkomplexen. ${ }^{19}$ Der Punkt ist nicht die Entwicklung von Technik (im Sinne von praktisch, intellektuell und ggf. emotions-beeinflussend ${ }^{20}$ Werkzeughaftem), denn das gab es immer und charakterisiert als solches noch keinen Äon. Doch die heute sich einrichtenden Gegebenheiten müssten erstmals von der Technik her begriffen werden, weil diese im Übergang in ein strukturell neuartiges, verabsolutiertes Stadium sei - das Zeitalter der Apparatewelt. ") Leben bedeutet [heute], Apparate zu füttern und von ihnen gefüttert zu werden« ${ }^{21}$

Die Menschen leben immer, zu allen Zeiten, in Vorstellungen. In eigenen geformten BilderVorstellungen innerhalb dessen bemächtigen sie sich der Wirklichkeit. Mit dem Erwachen und der Kultivierung ihrer eignen Kräfte dann beginnen sie, diese Vorstellungen - und darüber die Wirklichkeit - auszulegen und geistig anzueignen, auf einzelne Merkmalszüge hin, einzelne Bedingtheiten, Varianz, Gestaltbarkeit. Symbolisches Medium dessen ist leitend die Schrift und die mit ihr mögliche Produktion objektivierter Texte. Schrift ist symbolisches Medium, das sich als solches auch weiß. Im Ineinander und konkret Ringen zwischen Bildern und Schrift verläuft die kulturelle Entwicklung der Menschheit.

Die in Heraufkunft befindliche Spätphase der Moderne ist so der zweite große kulturelle Epochen-Umbruch. Der erste war jene Erfindung der Schrift gewesen, Bemächtigung mittels der Schrift. Sie bricht den Bann der Bilder, deren Holismus - die reine Oberfläche, opak, Zirkularität des Unveränderlichen, flächige Vereinnahmung des Geistes - den Menschen, obwohl selbstgeschaffen, gefesselt gehalten hatte. Die (mythischen) Bilder waren die primäre - »magische « - Besitzergreifung der Welt gewesen, hatten aber ihrerseits eine als mit Macht besetzte eidos-bzw. archetypos-Wirklichkeit zur magischen Norm reifiziert. Gegen dies eröffnete die Schrift und das mit ihr gestiftete lineare Denken Freiheitsdistanzen der rationalen Durchdringung (»Begreifen«). Mit dem Geist der Schrift verbunden sieht Flusser darum den Geist der 〉Erklärung ‘: die Welt bestehend aus einzelnen Kausal-

16 Vgl. G. Deleuze, Différence et répétition, Paris 1968; J. Baudrillard, L'échange symbolique et la mort, Paris 1976; ders., Les stratégies fatales, Paris 1983; ders., Simulacres et simulation, Paris 1985; P. Virilio, L'Horizon négatif: essai de dromoscopie, Paris 1984; ders., L'Inertie polaire, Paris 1990; und vieles mehr.

17 Bis hin zur Simulation der menschlichen Stimme und Simulation von von Menschen gespielten Musikinstrumenten.

18 In ihren positiven Möglichkeiten - als das Nicht-mehr-Hierarchische und statt dessen Vernetzte, als das Freiwerden zum Versuchsweise-Spielerischen und im Ganzen als Utopie des (allseits durchlässigen) Informationellen - von Flusser »telematische Gesellschaft« genannt.

19 Zum ganzen Folgenden: V. Flusser, Für eine Philosophie der Fotografie, Göttingen 1983 (zit. nach der überarbeiteten 5. Aufl. 1991); sowie ders., Ins Universum der technischen Bilder, Göttingen 1985.

20 Meditations-Techniken, Liebes-Techniken, >Effekt<-Techniken der künstlerischen Wirkung ...

21 V. Flusser, Für eine Philosophie der Fotografie, a. a. O., 72. 
Abhängigkeiten, die, obzwar verschlungenes Geflecht, sich wie eine Schrift lesen ließen - eine in vielerlei Linien Lesbarkeit der Wirklichkeit.

Am Ringen zwischen Bild und Schrift entscheidet sich so das Verhältnis von vereinnahmenden Vorstellungen des `Es-ist-so < und gewonnenen Erklärungs-Potentialen, aus welchen Szenarien eines Anders-sein-Könnens und praktisch ein >Ich-könnte...< aufscheinen. Das Zeitalter der Schrift ist denn auch das Zeitalter der Geschichte: Zeit der menschlichen Gestaltung der Welt - objektiv wie zugleich als Selbstverständnis dieser Potenz -, Epoche der immanenten Intentionalitäten. Sein Ende habe es sich selbst bereitet, mit einer Überproduktion von Texten, immer größerer Abstraktheit des Textsymbolischen und der Erklärungs-Systeme und mit zunehmender Selbstreferentialität - Verlust der Vorstellbarkeit. - Am Beispiel von exemplarisch der Fotografie zeigt sich für Flusser die hier gerade aus dem »Begreifen« - technischer (Konstruktion-) Idee zum zuhandenen Kulturinstrument dafür - geborene neue Bildlichkeit. Eine Wiederkehr der Bilder bringt das abstrakt (und gesellschaftlich elitär) gewordene Denken wieder zur Vorstellung. Doch eignet diesen technischen, d. h. mittels Apparaten generierten Bildern in dieser Funktion gerade eine täuschende Objektivität.

Mit der Fotografie, als neuer Errungenschaft des technischen Zeitalters, scheint die Wirklichkeit direkt vorstellungshaft-abbildlich gegeben. Damit aber vollstreckt sie zunächst und im Allgemeinen vielmehr nun auch in der Vorstellungswelt die Macht der inzwischen zur Herrschaft gekommenen Apparate; der Fotoapparat steht hier zusammen mit dem Verwaltungs-Apparat, dem Zahlen-verarbeitenden-Apparat (in den elektronischen Chips) oder dem >Nachrichten $<-A p p a r a t$ (der Medien). ${ }^{22}$ Die wahre Formung geschieht durch ein Programm sin ihres Mechanismus) in seiner Einrichtung darauf ausgerichtet ist, etwas wie die Unmittelbarkeit des >Es-ist-so< aussehen zu machen. Der Apparat generiert die Wirklichkeiten, aber der Apparat ist immer weitergehend eine black box - ein der Einsicht und dem Eingriff entzogenes hineinprogrammiertes Inneres, zwischen Bedien-Input und Erscheinens-Output, der sich in die Menge der schon bestehenden 〉Es-ist-so<-Outputs dieses Apparats einreiht.

Das verändert auch das Denken und die Erfahrung insgesamt. Statt der Kausalität, statt des Erklärbarkeits- und Interventionsbewusstseins, steht nun wieder eine Zirkularität, freilich die nach >ökonomischen` Parametern - solch Parametern wie der ökonomische Prozess - sich vollziehende Zirkularität der Umwälzung und Umgruppierung der Bedeutungen und der immanenten punktuellen Steigerungs-Neuheit: Bedeutungsgenerierung, die durch den dichten Kosmos der schon bestehenden herrschenden Bedeutungen und zugleich das Programm des Apparats fast total vorgegeben ist. Es ist ein Denken und Wahrnehmen zunehmend in den (Agierens-) Möglichkeiten des Apparats. Und es entsteht eine Ent-Dinglichung der Wirklichkeit; maßgebliche Wirklichkeit sind nur noch

$22 »$ Die Kamera [ist der] Ahne all jener Apparate [...], welche daran sind, alle unsere Lebensaspekte, von der äußeren Geste bis in das Innerste des Denkens, Fühlens und Wollens zu robotisieren.« (Für eine Philosophie der Fotografie, a. a. O., 64 f.) - Was die Ursachen des Umbruchs sind, so lokalisiert das Flusser'sche Modell sie denn genau besehen in zwei einander sich überlagernden Entwicklungen. Zum Einen in einem neuen Bedürfnis nach (vereinfachender) Bildlichkeit; gerade der Erfolg der graphischen Symbolsysteme (»Schrift« und die daraus hervorgegangenen logischen und wissenschaftlichen Formalkalküle) habe zu ihrem Niedergang als Mittel der allgemeinen sozialen Verständigung geführt, die vielmehr wieder Bilder brauche. Zum Anderen in der Entwicklung, wie - statt in aller früheren Geschichte allein »Werkzeuge« - aus dem »begreifenden Denken« mit der industriellen Entwicklung zunächst Maschinen in die Arbeits- und Lebenswelt gekommen sind, bei denen bereits die Umkehrung des Handlungsblicks eintritt: dass der Mensch sich der Maschine anpasst, den Parametern zu deren Handhabung und Rhythmus; und wie - parallel zu dem, dass die industrielle Herstellung von Güter an (gesellschaftlicher) Bedeutung verliert zugunsten der Schaffung und des Austauschs symbolischer Bedeutungen - »nachindustriell« aus den Maschinen Apparate, ein System von Apparaten, geworden sind. 
Botschaften, Information und vor allem Wie-Information-Aussehen, Im-Universum-der-Informationen-Erscheinen. -

Die Theorie von Flusser darf als exemplarisch gelten für die Bemühungen der Gegenwart, den Umbruch in der Moderne, der sich in den heutigen Erfahrungen der Wirklichkeit spiegelt, in seiner Epochalität zu begreifen. Ihre konzeptionelle Überlegung ist: Technik. An den Phänomenen und Auswirkungen der Technik in der bestehenden Welt bekunde sich die Grenzscheide einer neuen Epoche. Gegen die Tradition bisherigen Geschichts- und Kulturdenkens, zeigen sie Technik als einen eigenen Faktor und eigene Macht, die das Wesen der heutigen Welt mitbestimmt, zu reflektieren. Intentionalitäts-Verständnisse der sozialen Wirklichkeit - auch wo kollektive oder >anonyme` (resp. >maskierte $<$ ) Subjekte - hätten dies verdeckt, das entstandene Neue und die Macht gerade auch in Ausstrahlung auf unser Denken, die Kultur und die Form des Gesellschaftlichen. Das Gestaltstadium des Technischen - bei Flusser: die zum »Apparat« gewordenen (Erfindungen von) Maschinen - lasse sich nicht bzw. nicht mehr in der Linie des menschlichen Werkzeug-Gebrauchs, als dessen eben akkumulierte Steigerung, modellieren oder als in der Nachfolge des Prometheischen Feuerraubs. Das der Verdacht gegen den Schatten der >philosophischen` Ideologie der Moderne, ihre >Subjekt<-Ideologie.

Das Motiv der Theorie Flussers bleibt dabei das einer Kulturkritik - in dem Bewusstsein, dass es dafür einer neuen Form von Kulturkritik bedarf. Diese differenziert, aus zeitbedingt-strategischen Gründen, sozialwissenschaftliche und philosophische Dimension. Es ist im Endeffekt eine gedankliche (theoretische) Polarisierung zwischen beidem. Eine nüchterne sozialwissenschaftliche Diagnose $^{23}$ zeigt, wie zunehmend die Monopole - und damit auch die genuinen Erfahrungen - des menschlichen Aktors sich auflösen; wie einstmals menschlich Getätigtes durch ein in einen Apparat eingepflanztes Programm ersetzt wird. In einer Welt der Apparate, mit denen umzugehen ist, transformiert sich alles zu einer nur noch funktionalen Verhaltensweise und funktionalen Erklärungen auch. Der immense Kosmos des herangewachsenen Kausal-Begreifens, der Apparate-Ideen und überhaupt des kulturellen Stands, der - dessen ganze Hierarchie - in den Apparat implementiert ist und den Menschen zum »Funktionär« des (betreffenden) Apparats bzw. der Apparate-Möglichkeiten formt, wird unsichtbar. Das Zeitalter wird kritiklos. Alle Impulse des Kritischen, die modern aus den Erfahrungen der Wirklichkeit erwuchsen, sind schon je vom Apparat absorbiert. >Humanistische Anklage (à la `Entfremdung`, ১Unfreiheit`, >Klassen-Ausbeutung` oder `falsches Bewusstsein`) greift mehr und mehr ins Leere.

Das dynamische Verhältnis von Bild und Schrift ist in ein neues Bilder-Verhalten übergegangen. Im Egalitarismus der Bilder, allgemein: der durch Handhabung ausgelösten Output-Zeigungen, scheint jeder Außenpol und jede nicht schon in das Gefüge eingepasste Erfahrung verschwunden. Und doch - stehe es als die Aufgabe einer Philosophie der Phänomene, Sachwalter des fast schon Unmöglichen zu sein: den Kampf weniger Einzelner in der Totalität des Bilder-Universums zu denken und theoretisch zu flankieren. Als letzte noch bleibende Position stehe die Philosophie in der intellektuellen Pflicht des Humanen, den historischen Blick festzuhalten - und die kleinen Lücken in der Totalität bewusst zu halten, die kleinen Fugen der Freiheit, dem Apparat das Nicht-Vorgesehene abzuringen, mit dem Apparat gegen den Apparat, als Symbol oder Vorschein eines Anders-Seins. ${ }^{24}$

\section{Der Zustand der Welt und das Werden zum Subjekt}

Die Diagnose Flussers scheint heute nicht mehr besonders aufwühlend. Unsere Gegenwart hat sich an Endzeit-Szenarien gewöhnt. Dass in kaum zweieinhalb Jahrzehnten das schlichtweg Irrelevant-

23 Vom konzeptionellen Typus her: medientheoretisch.

24 Vgl. V. Flusser, Für eine Philosophie der Fotografie, a. a. O., 68-74 (auch 62-68: gegen alle bisherigen klassischen Muster der Kritik); »historisches « = »kritisches Bewusstsein«: 56-58. 
Werden aller Ideen und Selbstverständnisse der Moderne ins normale Bewusstsein eingegangen ist, ist Index des Einschneidenden der Wandlungen.

Mit einem gewissen Achselzucken vor dieser Diagnose zu stehen hat freilich auch Ursachen in deren theoretischem Profil selbst. Es sind Betrachtungen der objektiven Kultur. Die subjektseitige Spezifik der Moderne - und dann Pol ihres Driftens in die »Nachgeschichte $\ll^{25}-$ ist gefasst nur als generell Begreifen, Erklärungsrationalität, technische Rationalität; ${ }^{26}$ und dabei eine Moderne, die im Prinzip schon in der griechischen Antike beginne, der Weg zum logos. Das Soziale kommt gar nicht vor; und die Dimension des Selbstverständnisses ist übersprungen, eingeschmolzen in das praktische Haben der Welt, in einem symbolischen Praktizismus. Es sind Betrachtungen auf der Basis eines undifferenziert-universellen Verständnisses von menschlichem Selbst und Person-Sein - eben, Subjektivität >gibt es` (Welt-Wahrnehmen, Bedürfnisse/Zwecke, Werkzeug-Verhalten, Herstellen), und dieses Humane sei heute von den Apparaten aufgesogen bzw. ausgetrocknet.

Die Tragweite dessen, was Wirklichkeiten bedeuten für die menschlichen Wesen in ihnen, ist in weit erfahrungsgenauerem Problembewusstsein vielmehr von der heute allzu epochal verfemten philosophischen Tradition gedacht. Am entschiedensten dies bei Hegel. Von Hegel aus kann in ungleich tieferer Differenziertheit in den Blick kommen, wie die Rationalitäts-Verkörperungen einer herrschenden Wirklichkeit - die Welt immer dichter werdend besetzt von Gegenständen der Rationalität - auf die Profilgestalt der Subjektivität in sich, die Selbstverständnisse wie Selbstbilder, zurückwirken. In heutigen Thematisierungen wie exemplarisch der von Flusser dagegen ist das Problem verharmlost und zugleich dramatisiert. Die Perspektive - Modellierung - ist im Grunde nur 1-schichtig. Zudem hat sie bezeichnenderweise kein Sensorium für die parallele Entwicklung im Bereich des Wissens, ${ }^{27}$ dass genauso wie Veränderungen in einer Welt der Apparate auch das allseitige Umgebensein durch Bestände von >Wissen « den Erfahrungsraum der Subjekte einschneidend umformt.

Mit Hegel lässt sich verstehen, wie ein Zustand der Wirklichkeit auch ein soziales Selbstverständnis induziert. Hegel zeigt Moderne als gekennzeichnet durch einen qualitativen Begriff von SubjektSein - einen bestimmten Sozialbegriff von Subjektivität. Entfaltet hat er dies charakteristisch für den Anfang, das Urgeschehen einer in die >Moderneく eintretenden, für >Moderneく offen werdenden Gesellschaft, und wohl gerade deshalb hat er den Kern der Zusammenhänge weit deutlicher bezeichnet als heutige Theorien, die, von einem Globalbegriff `moderner Subjektivität ‘ ausgehend, ganz auf den finalen Umbruch oder Kollaps fixiert sind. Erstmals in dem ehedem von Rosenkranz - unter der Einschätzung, hier das ineins religions- wie geschichtsphilosophische Schlussstück (»Fortsetzung«) des Systems der Sittlichkeit vor sich zu haben - mitgeteilten Text ausgebreitet, und dann aber auch in Hegels späterer Philosophie der Geschichte sowie vielfachen parallelen Zusammenhängen, findet sich der Gedanke illustriert, dass das, was den Prozess der Moderne und seinen Gang verstehen lässt, nicht einfach in der Universalität der neuzeitlichen Vernunft zu lokalisieren ist, desgleichen nicht erst in der materialen Kultur des Rationalen und den geschaffenen Rationalitätsgebilden besteht - mithin als eine Veränderung im Intellektualen, Entstehung eines vernunft-intellektuellen Selbstverständnisses und der dadurch in die Welt gekommenen Folgen. Am entscheidenden und für das Verstehen

25 Vgl. V. Flusser, Für eine Philosophie der Fotografie, a. a. O., 70 f.; ders., Nachgeschichte. Eine korrigierte Geschichtsschreibung [Schriften in 9 Bänden, Band 2], Bensheim [u. a.] 1993.

26 Die Problematik - zu geringe Differenzierungstiefe - beginnt schon damit, dass dieser heutige Theoriezugang ein $z u$ 〉klassisches〈 Konzept von »Werkzeug«, als Abhebungsfolie für das epochale Novum der Apparatewelt, ansetzt: rein intentionalistisch, im Grunde eine krude >anthropologische< Technik-Theorie in der Tradition des ersten Begründers E. Kapp (vgl. Grundlinien einer Philosophie der Technik, Braunschweig 1877).

27 Bei Flusser dies wohl vor allem wegen seines Begriffs von »Bild«: ein entschränkt weiter Begriff, und weithin undifferenziert. Umgekehrt denken Theorien wie etwa Baudrillard usw. die - gleicherweise nur objektive Kultur allein als Symbolisches, gar nicht die Materialität, die sie auch ist. 
alles Weiteren bis zur heutigen Spätentwicklung strukturorientierenden Beginn steht für Hegel vielmehr der Weltzustand, der die Einzelnen zu: >Personen ‘ geformt hat und ihre Bildungen-des-Sozialen zu: Verhältnissen zwischen - willens-haften, sich in individuellen Interessen einrichtenden, sich mit einem Feld (»Sphäre«) der 〉Sachen des je Ihrigen identifizierenden - >Personen〈. Also der Weltzustand, für den historisch das Römische Reich steht und die Geltungsprinzipien seiner Ordnung. ${ }^{28}$

>Person` (und die je ihrigen `Sachen`, in die sie sich berechtigterweise, d.h. ohne Anmaßung sowie ohne Übergrifflichkeiten in betreffende Felder Anderer, verlegt hat): das ist das, was in der Ordnung gilt; ist, was in den (etabliert-herrschenden) Praxen, Handlungsweisen, dem Umgehen mit Kausal-Beherrschung, mit Welt-> Wissen « und mit zuhandenen Gerätschaften, und was nicht zuletzt in dem, wie man einander gegenüber sich verhält, einander behandelt - was darin allem als das zugehörige `wahre ( (und zu achtende) Subjekt verstanden ist. Das Novum dieses Weltzustands aber ist nach Hegels Einsicht nur die eine Hälfte. Denn in diesem Äußeren, das alle vorgegebene äußerliche Besonderheit, Besonderheit von Geburt, Geschlecht, Ethnie, Status, mit seiner Macht nivelliert hat - ein jeder zunächst formalermaßen gleicherweise `Person $\prec-$, wurde Raum für ein Inneres der Besonderheit im Geistigen, des besonderen Werts der Individualität: nach Hegels geschichtsphilosophischer Deutung bekanntlich der Boden, auf dem dann das Christentum Resonanz finden und sich ausbreiten konnte - als das Selbstkonzept des Inneren für dieses Äußere, das Äußere der WeltOrdnung der $>$ Personen $<{ }^{29}$

Mit der heutigen Zuspitzung, heutigen Zustand der Moderne hat sich dieses Verhältnis von >Person` und authentischer Subjektivität - plakativ: das von Hegel am Zusammenhang von gesellschaftlich-politischer Normordnung der römischen Welt und christlicher Innerlichkeit illustrierte Verhältnis - radikal gewandelt. Es ist gekippt in eine Dynamik der Selbstaushöhlung und zugleich des Extremismus. - Ein letzter Abschnitt soll der theoretischen Bewandtnis dieses Zusammenhangs, und theoretischen Folgerungen, gelten.

\section{IV.Das Suchen nach dem wahrhaften Subjekt (Negativität ohne Folgen)}

\section{Im Stand der Persönlichkeit}

Hobbes hatte eine Unterscheidung eingeführt in den neuzeitlichen Begriff der Subjektivität, und Hegel, seit Naturrechts-Aufsatz und System der Sittlichkeit des epochengeschichtlichen Erfahrungsgehalts bewusst, der sich im Hobbes'schen radikalen empiristischen Denken ausspricht, ${ }^{30}$ hat sie aus der Hobbes-Tradition des >Person<-Begriffs aufgenommen. $\mathrm{Zu}$ den zentralen, wiewohl äußerlich fast versteckten Einsichten von Hobbes gehörte, zwischen natürlichem Menschen - seinen Regungen, Interessen, Gefährdungen - und dem großen über allem thronenden Leviathan - der absoluten Schicksalsmacht unserer irdischen Sozialexistenz - als gleichsam Drittes, Vermittelndes das sozialöffentliche Gelten-als-... zu markieren: die geltende und agierende Persona, die im Namen

28 Vgl. K. Rosenkranz, G. W. F. Hegels Leben, Berlin 1844, 132-141 (jetzt in G. W. F. Hegel, Gesammelte Werke, Hamburg 1968 ff., Bd. 5, 459-465); G. W. F. Hegel, Vorlesungen über die Philosophie der Weltgeschichte, Bd. 1: Die Vernunft in der Geschichte, Hamburg 1955, 250-257; ders., Werke in zwanzig Bänden, Frankfurt/M. 1970 ff., Bd. 12, 380-406.

29 Vgl. ebd.

30 Als das Recht des Empirischen (empirischen Phänomendimension in der philosophischen Wissenschaft) gegen den konzeptionellen Formalismus des neueren Denkens, wie er in Kant und Fichte seine exemplarische Zuspitzung erfahren habe: vgl. Hegel, Gesammelte Werke, a. a. O., Bd. 4, 420-430; Bd. 5, 281-295. 
eines Interessensubjekts handelt oder spricht. Dieses Subjekt ist entweder die auftretende Person selbst, in ihrer ganzen eigenen Natürlichkeit, die dahinter steht; oder aber - im gesellschaftlichen Zustand - vielfach ein Anderer (oder andere Instanz), für den ich stehe, dem ich mich verschrieben habe, der mich seine Autorschaft zu der meinen hat machen lassen: mich dazu ermächtigt oder umgekehrt dazu geformt hat. Im Sozialzustand agieren und reden viele solche »künstliche Personen«, deren eigentliches autorisierend-vertretenes Subjekt - das Für-wen, Statt-wessen, In-welcher-Sache - normalerweise nicht als solches anwesend ist, nicht offen mitagiert im Raum des Anwesenden oder mitspricht. ${ }^{31}$ Die »künstlichen Personen « werden erst durch die Geltungen des herrschenden Sozialzustands, der >Person`-Ordnung, zu Stimme und Autorität im Geschehen der Interaktion, eigenen Polen der Handlungs- und Statuierungswelt. >Person` zu sein ist dabei auch ein etabliertes Vertrauen: ein - aus Alltags-Erfahrungen erwachsenes - Vertrauen in das berechtigte Subjekt dahinter und in die Wege der Autorisierung.

Hobbes hatte diesen Zusammenhang als Strukturbedingung des sozialen Zustands gezeichnet, gewissermaßen nur als universelles unabdingbares Moment bzw. Dimension jedes höheren Sozialen. Mit dem >geschichtsphilosophischen〈 Deutungsansatz Hegels aus der »Fortsetzung « des Systems der Sittlichkeit, der Philosophie der Geschichte und den anderen parallelen Theoriethematisierungen $^{32}$ demgegenüber lässt sich der Zusammenhang als Prozess, und in seiner Dynamik des >Modernen`, begreifen. Es ist ein Prozess der Formung der natürlichen Menschen $z u$ >Personen (und `Person〈-Verhältnissen) - dass sie selber wie >Personen` agieren und sprechen, d. h. den Standards des herrschenden Zustands gemäßerweise, und dass sie das persona-hafte Agieren-und-Sprechen Anderer als Wie-ein-Mensch nehmen und gelten lassen. Hegel spricht von »Zucht der Welt« u. dgl.: »die Individuen [müssen] in den Stand der Persönlichkeit treten«, »zur Allgemeinheit gereinigt ${ }^{33}$ - durch eine Wirklichkeit zu einem bestimmten Subjektivitäts-Charakter geformt zu werden. Und das meint, diese Wirklichkeit durchaus nicht nur als die kursierenden Werte und Bewertungen und die thematisierende Reflexion-über-Subjektivität, also geistig-ideelle Wirklichkeit, an mich kommende >kulturelle< Tradition. Sondern, so Hegels Konzept von »objektiver Geist« und »sittlicher Welt«, Wirklichkeit allem voran als das Entgegenstehende, das Erfahrbare und Macht des Erfahren-Müssens: Wirklichkeit qua die Art der Dinge, mit denen man umzugehen hat; die Weisen von Handlungen und Verhalten; mein Behandelt-Werden; die handgreiflichen Handlungsfolgen sowie entsprechenden Handlungs-Erklärungen. Immer, von dem historischen Urgeschehen der Stiftung genauer: Erzwingung - einer öffentlichen Allgemeinheit an, die mit dem Zustand 〉Rom gekommen ist und einen Standard behauptungsmächtiger Ordnung gibt, sind die natürlichen Besonderheiten nicht nur: >Personen`. Sondern es bilden sich in den Einzelnen bestimmte Bilder, was sie

31 So in einem kleinen Übergangskapitel im Leviathan (Chapter 16 >Of Persons, Authors, and things Personated $\measuredangle$ ) und gleicherweise in den Elementen der Philosophie (Elementorum philosophiae sectio secunda: De homine, Kap. $15>$ De homine Fictitio, sive de Persona $)$. Hobbes spricht von »Feigned or Artificall person[s] « (Leviathan, ed. C. B. Macpherson, London 1968, 217). - Vgl. meinen Aufsatz »Persona. Über das stellvertretende Leben«, in: K. Röttgers, M. Schmitz-Emans (Hg.), Masken, Essen 2009, 97-131.

32 Was darunter die Thematisierung innerhalb der Rechtsphilosophie betrifft, so ist dort die spezielle, durch den Theoriekontext bedingte Begriffsverwendung zu beachten, in der »Person« und »Subjekt « - beides freilich als »Geistiges«, strukturell anders als bei Hobbes - in überhaupt eine prinzipielle, dagegen nicht vornehmlich geschichtlich-dynamische Polarisierung kommen (»Person« qua Träger von Rechten und »Subjekt« als Wirklichkeit der »Moralität«/ moralischen Reflexion). Vgl. Grundlinien der Philosophie des Rechts, §§ 35 (plus Zus.), 105, 113 Zus., 123 Zus.

33 »In den Stand der Persönlichkeit treten«: analog wie in den Stand der Ehegemeinschaft, zur Konstituierung der elementaren sozialen Einheiten des Gesellschaftlichen. - Zitate: Die Vernunft in der Geschichte, a. a. O., 119, 253; Werke in zwanzig Bänden, a. a. O., 388. 
als die darin Agierenden und Sprechenden, was als >Personen ausmache. ${ }^{34}$ Es sind Innen-Bilder, die sich, durch Erfahrungen, an der Außen-Wirklichkeit ${ }^{35}$ herausformen. Im anfänglichen Zustand, dem Zustand des historischen Römischen Reichs, Innen-Bilder des freigegebenen privaten Sinnens und Trachtens - anerkannt zu sein darin, in Willkürfreiheit mich in die Sachen des Meinigen zu verlegen; doch mit der Erfahrung des Zwiespalts zwischen Weltlichem und Geistigem - welches geistige Bewusstsein mit dem Christentum zu Idee und Ideal einer positiven Selbstergreifung und Kultivierung gekommen ist - sowie der langen geschichtlichen Arbeit an deren Versöhnung ein zunehmend qualitativ-geistiges Innen-Bild.

So ist denn auch >die Persönlichkeit der Moderne`, so möchte ich die Hegelschen Einsichten nehmen, nicht nur: der »vernünftige« Mensch, der wissende, reflektierte, seine »Moralität« geltend machende, tendenziell traditionsautoritäts- und kirchen-skeptische, der zu Gefühlsäußerungen und Selbstpsychologisierung fähige Mensch, als der er sein Auftreten hat. Sondern es ist auch eine epochale >Person<-Deutung, bestimmte >Persönlichkeits $<-$ Struktur, >Persönlichkeits $<-P r o f i l$, dynamisches Verhältnis von >Person` (persona) und Subjektivität-ihres->Ichく. Mit der Neuzeit, mit Entdeckungsreisen (Welt-Eroberung) und Reformation, mit wissenschaftlicher Erkenntnis (neuen Wissenschaften) und cartesianischer Philosophie ${ }^{36}$ ist das geistige Bewusstsein in allgemeine Wirklichkeit eingegangen, hat sich in die Wirklichkeit der Handlungswelt und Welt des Gültigen-Wissens eingeschrieben, so dass diese Verhältnis-Struktur eine bestehende Substantialität gewonnen hat. Nach der großen Entwicklung des Ausgleichs von Weltlichem und Geistigem sind `Person<-Sein und Ich-Authentizität nun beidenteils Phänomene zweier Seiten - etablierte >Person<-Welt und subjektives >Person<-Verständnis, sowie Gewissheit meiner Selbst und diese Subjektivitäts-Welt, individuelle Innenorientierung meiner Überzeugungen und Wichtigkeiten, als ihrerseits sozial geltend, soziale Wirklichkeit. Das erfordert eine weitere, letzte Entscheidung im Bau der Theorie.

\section{Abwesenheiten}

Der von Hegel gesehene Prozess von >Person« und Subjekt-Ich besitzt eine in Hegels Konzept des »Geistes« eher nur implizierte, nicht material ausbuchstabierte Dimension, aus der indes Wesentliches seines historisch-analytischen Potentials resultiert, bes. sein Potential für die Phänomene des Heutigen. ${ }^{37}$ Denn zu der Entwicklung des Doppelzusammenhangs von >Person` und Subjekt gehört, als das Werden einer humanen sozialen Welt dessen, offenkundig der Gleichtakt der Herausgestaltungen von beidem, der Abgleich zwischen Akteurs- bzw. Sprecher-Figur (genauer: Figurformen) und autorisierendem Wer, das Vermittelnde der empirischen Erfahrungen des Stehens-für-... - jenes von Hobbes erkannte zugehörige Vertrauen in das berechtigte Subjekt dahinter und in die Wege der Autorisierung. Nicht nur herrschend-verbreitete und geübte kulturelle Muster, kulturelle >Traditionen`, sondern wesentlich auch die Wirklichkeiten von Handeln und Wissensformen - `Gegenstände`, Bedingungen, Können sowie Typen-des-Wie - formen Dispositionen und Erwartungen, ${ }^{38}$ die jeweiligen Innen-Bilder in den Subjekten auch durch ihr eignes (mit-machendes) Sich-Geben verstetigt. In ihrer sozial vorherrschenden Habitualität bilden sie, über die Wirklichkeiten der ge-

34 Damit verwoben entscheidend auch das Negativprofil: Vorstellungen, wo bzw. wie sie ihre Subjektivität verfehlen, wo ihrer Persönlichkeit und Souveränität verlustig gehen.

35 Für Hegel bes. der der Handlungswelt.

36 Hinzu - bei Hegel selber nicht so eigens herausgestellt - den sozialen Bewegungen der Freiheit.

37 Weit mehr noch als für Flusser gilt für Hegel, dass die Tiefe seiner Theorie darin sich beweist, Entwicklungen im Prozess der Moderne, die zu seiner Zeit in ihren konkreten lebensweltlichen Ausprägungen noch nicht im Mindesten vor Augen lagen oder sich andeuteten, im Strukturellen der aufkommenden Veränderungen begreifen zu lassen, wenn nicht gar selber vorausgesehen oder doch vorgezeichnet zu haben.

38 Angefangen bei den Horizonten, was als \Situation` verstanden ist. 
sellschaftlichen Stratifikation, Zugang zu Gütern, Chancen auf stadiumsadäquate Qualifikation usw. (s. o.) hinaus, soziale Lebenswirklichkeiten zweiter Stufe, und diese bedürfen, um der humanen Gestalt der prozessierten Interaktion in Handeln und Wissen willen, der Erfahrbarkeit der Perso$n a$ - und Subjekt-Zuordnungen: im ausgeglichenen Zustand, lebensweltlichen Abgleich zwischen Akteurs- bzw. Sprecher-Figur (Figurformen) und autorisierendem Wer, eine zweite Natur sowohl der bestehenden Welt wie zweite Natur des Subjekts. ${ }^{39}$

Doch wenn die Ketten des Stehens-für-... abreißen - und wenn die Masse der uns in einer bestimmten Weise fordernden und zugleich undurchschaubaren Handhabungs-Gegenständlichkeiten des Praktischen wie Bestände zu wissenden Wissens zu übergroß geworden ist -, gerät die Macht unserer >Personen in Krise. Es erwachsen krisenhafte Subjekt-Imaginationen und Subjekt-Projektionen, während zugleich das Spiel der Personae läuft und weiterläuft und durch unser Mit-Machen wiederum auf die Selbstverständnisse und Verständnisse von >Situation`zurückwirkt. Man sollte dies sicher nicht gleich so hoch hängen, von >falschem Bewusstsein u. dgl. zu reden. Doch ist es etwas, das, als diese subjektseitige Dimension, die anomischen Tendenzen des Gesellschaftlichen nur noch weiter anheizt, die Mechanismen der Integration sabotiert. Nicht nur, dass an den erfahrenen negativen Wirklichkeiten im Zuge der Modernisierung (s. o. II.) keine adäquaten, die Widersprüche übergreifenden Verständnisse des bewirkenden Warum mehr möglich sind, Verständnisse der Autorschaft und intentionalen Bewusstseinshorizonte (»Sinn «; durch-irgendeine-Instanz-bezweckt-oderverursacht). Sondern zunehmend ist überhaupt der Prozess der Bildung von Selbstverständnissen unterlaufen.

Zur Krise gehören ihre Symptome. Wesentliche der Ausprägungen - der mentalen Ausprägungen - unseres Zustands der >Moderne lassen sich als Effekte dieser Rückwirkungen der Wirklichkeiten auf die Innenstrukturen des Selbst verstehen. Sie reichen von der Faszination des aus dem Weiten der Welt - der zunehmenden Universalität von Technik, Wissenschaft, Alltagskultur und Lebensstil - kommenden >Neuen` und >Neuesten` (und der dadurch bewirkten Überblendung, ja Verdrängung des Alten) bis zur Wiederkehr des gerade Lokalen in diesen $z u$ groß gewordenen Räumen; von der Sehnsucht nach dem Du bis zu den Empfindungen, den Anderen zugleich als Bedrängung und Invasion zu erleben und sich gegen Übergrifflichkeiten, Instrumentalisiert- und Ausgesaugtwerden schützen zu müssen; von den Lockungen des Aufgehens, des Verschwindens in der Gemeinschaft - ein Sich-Spüren als Teil einer Masse (auch >Trend -Masse) - bis zum Hunger nach authentischen Erlebnissen; vom empfundenen eigenen Erkalten - dem Verlangen nach dem verloren

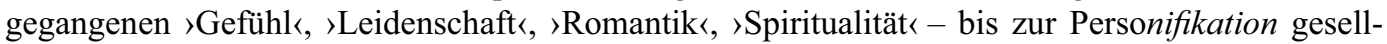
schaftlicher Mechanismen und struktureller Entwicklungen; von erpressten Stellvertretungen - an Stelle eines ausfallenden oder sich entziehenden Anderen das humane Gesicht zu bezeugen - bis zur Selbstbezichtigung, dem (tiefenpsychologischen) Verdacht gegen die Mächte meines eignen Unbewussten; von einem allseitigen Bekennertum und Entblößung (selbst des Peinlichen und Intimsten), auch Auflösung aller Schamesschwellen, bis zum neuen cocooning, der Einhausung in vermeintlich sichere Räume des Persönlichen und Privaten, Zurücktreten vom Bürger- und Gemeinschaftsbewusstsein; von der Suche nach wahrer Ernsthaftigkeit und nach innerer Sammlung bis zu den immer größeren und selbstverständlicheren Bedürfnissen, im Alltag \Spass $</>f u n<$ und entspannende Zerstreuung zuteil zu bekommen (bei immer geringerer Frustrationsschwelle, falls nicht bzw. nicht genug); vom Glauben an die große Ratio evolutionärer Prozesse - das Bestehend-Herrschende als das, weil angepasst-an-die-Bedingungen, Sich-durchgesetzt-Habende - bis zur neuen Metaphysik der >Tragik und des >Rätsels ‘ allen Seins; vom Rückzug der Wissenschaftlichkeit ins immer Forma-

39 Zwei klassische Bedeutungen von »zweiter Natur« kommen bei Hegel zusammen: sind miteinander verwoben als die zwei Seiten desselben Phänomenprozesses des Geistigen. Vgl. etwa Grundlinien der Philosophie des Rechts, $§ 4$ / § 151; Werke in zwanzig Bänden, a. a. O., Bd. 10, 183 ff. (Enzyklopädie, $\S 410)$; Bd. 12, 57; Bd. 17, 146. 
lere, in den Typus Strukturwissenschaft und universelle Begriffssprachen, bis zu den Projektionen von (verloren-einstiger, ursprünglicher oder erkenntnistranszendenter) 〉Fülleく, überhaupt generellen Ästhetisierungen von Phänomen- und Erfahrungsverständnissen, ästhetisierenden Terminologien wie Kriterien; ${ }^{40}$ von einer allgemeinen Virtualisierung von Autorschaften und Verantwortung bis zur Feier der Selbsterfindung, Flucht in ein neues Ich, 2nd life; und schließlich: von unbestimmten Unzufriedenheitspotentiale ${ }^{41}$ bis zur Absorbierung aller Unternehmungen prägnanter argumentierender Kritik, deren Impulse an der Elastizität und Zähigkeit des Bestehenden - dessen ungerührtem Weitergehen: das Herrschende smart und zugleich zynisch geworden - ins Resonanzlose zu laufen scheinen.

$\mathrm{Zu}$ den Ausprägungen der Moderne im heutigen Stadium des Zeitalters gehören so auch Entweltlichungen gerade in dem, was sie einst ausgemacht hatte: dem Wirklich-Werden der Subjektivität und ihres Rechts auf eignes Bedürfnis, eignes Erfüllungsempfinden und eigne Meinungs- wie Urteilswelt. Die Phänomene der gegenwärtigen Epoche bekunden das Dass des krisenhaft Gewordenen - dass die Selbst-Bilder in fressende Verformungen geraten sind und umgekehrt die Einzelnen sich nicht mehr erfüllend zu erleben vermögen in den Szenen der agierenden und sprechenden Personae, auch den von ihnen selbst verkörperten. In den bestehenden - und von jedem Einzelnen auch mit-exekutierten - Lebenswirklichkeiten kommt es, die Sinnerfahrungen auf immer begrenztere und fragmentarisierte Horizonte zurückgedrängt, offenkundig zur Auflösung überhaupt eines identitätstragenden Verständnisses menschlicher Handlung und auch menschlicher Kollision. Vom Werden der 〉Person`, Formung der Einzelnen - und Lernen am Bestehenden, durch Sozialisation - zur Persona von Agieren und Sprechen (Konstatieren, Urteilen) ist vor allem nur noch die Seite der EntWertung der natürlichen Besonderheiten machtvoll, ${ }^{42}$ Ent-Wertung der natürlichen Charakteristik und elementaren zwischenmenschlichen (Sozial-) Erfahrungen (sowie >Werten<), auch der daraus erwachsenden Widerständigkeiten, Sperrigkeiten. In einer Welt der geschaffenen Gerätschaften - in Handhabungsgegenständen geronnenen rationalen Ideen - und übervoll von Beständen von geltungsbeanspruchendem $>$ Wissen $<$ ist das System der mit den getätigten (und mit-gemachten) Praxen von Agieren und Sprechen transportierten Personae und 〉Person〈-Deutungen die Wirklichkeit. Und ist ein System, gegen dessen Vereinnahmung und Druck auf die Innenstrukturen des Selbst - die Innen-Bilder - nichts Eigenwertiges auf Subjekt-Seite unkorrumpiert Stand hält, realistisch, handlungsmächtig und potentiell distanzfähig. Dass genau die Frage, was uns als >Personen` ausmache, heute zu einem so zentralen Problem geworden ist, ist darum nicht nur durch die Verschiebung von Grenzen des `Lebens $\triangleleft$ - möglich gewordene technische (medizinische) und genetische Eingriffstiefe - und durch das immer nähere Emporwachsen `künstlicher Intelligenz`, sondern ist entscheidend mitbedingt durch das, was das Agieren und Sprechen in den Wirklichkeiten einer solch veränderten Lebenswelt bedeutet für die Füllung der Muster von `Person`-Sein und Subjekt, von Authentizität und Identitäts-Bedingungen.

Von dem, was sich in jenen realitätsentkoppelt gewordenen, flottierenden Selbst-Bildern niederschlägt, sind auch die Reflexion und die Theorie erfasst. Die verschiedenen und zum Teil so diskrepanten >Modernen` (gesellschaftliche, technische, ökonomische, ästhetische usw.), die die neuere Theorie zu identifizieren unternimmt, sind der Spiegel eines disparat gewordenen Zeitalters,

40 Dazu gehört auch, die Kunst ihrerseits zu einem (inter-) personalen Verhältnis werden zu lassen: als >Begegnungく, als >hat mich ergriffen` (nämlich weil sonst kein Spüren mehr) etc. Zuweilen mit Tendenzen zur Reauratisierung der Kunst, bei gleichzeitig zunehmendem Event-Charakter (sowie Bewusstsein der sehr profanen Mechanismen des Kunst-Markts).

41 Unzufriedenheitspotentialen, die, verwoben mit realen Unsicherheits- und Deklassierungsängsten, dann zu sozialen Vorurteilsmustern, Wut und Hassgefühlen medial bedient, ausgebeutet und angeheizt werden können.

42 So wie für Hegel im frühen Römischen Weltreich, vor Eintritt des Christentums. 
einer in die Erfahrungsräume hineingreifenden Pluralität der Entwicklung, Mächte und Tendenzen ohne fassbare Einheit. Wo am Ende des 19. und konzeptionell verstärkt in der ersten Hälfte des 20. Jahrhunderts die verschiedenen >personalistischen` Strömungen ein integrales Verständnis von humanem Selbst, geistiger Identität und authentischer Intersozialität zu retten versuchten in der realen Modernisierung ${ }^{43}$ - zugleich eine Sehnsucht nach menschlicher Unergründlichkeit und Unersetzbarkeit -, gibt es heute keine allgemeiner verbindlichen Bestimmungen mehr, was die Epoche geistigerweise immer war: ein Prozess begleitet von einer Thematisierung seines Wegs und seiner Ideale und eingreifender Kritik. Die alten Formen, das Versprechen auf menschliche Erfüllung, das Moderne stets war, einzuklagen gegen die Wirklichkeit und ihre Entwicklungen, greifen zusehends nicht mehr, ${ }^{44}$ und subjektiv sind die Orte der Beschädigungen, der gesellschaftsrealen und der mentalen, immer weniger identifizierbar. Statt dessen sind, neben Projektionen anonymer Mächte, die Diskonformitäten sich selber zugeschrieben; verbunden mit Ängsten, was passiere, wenn man sich dieser Moderne, den Faktizitäten der herrschenden Moderne, verweigere.

So ist die Wirklichkeit heute umstellt von Theorien: $z u$ vielen Theorien, nicht etwa zu wenigen. ${ }^{45}$ Die negative Stimmung bleibt, durch laute Fröhlichkeit allenfalls übertönt. Der Widerstand aber verschwindet. Dass die Epoche auf ihrem Weg der Moderne »nicht reich genug« ist, trifft so zuletzt auch für den Zusammenhang von Zeitalter und Kritik, Wirklichkeitszustand und Reflexion - begleitender kritischer Arbeit an ihrem Gang - zu: eine immer größere Unbestimmtheit, in welche Richtung das läge, dass die Einzelnen in den agierenden und sprechenden >Personen ২ der sozialen Welt sich wiederzuerkennen vermöchten. Das hieße, eine freie Gesellschaft.

\section{Rainer Adolphi}

Technische Universität Berlin

Institut für Philosophie (H 72)

Straße des 17. Juni 135

D-10623 Berlin

Email: rainer.adolphi@tu-berlin.de

43 Die breiten >personalistischen< Konzeptionen in Philosophie und philosophischen Wissenschaften sind mehr Symptom der beginnenden, bereits spürbar gewordenen Krise - wofür »Person«/»Personalität« alles einstehen soll in der Theorie -, als dass sie die Lösung wären oder doch zur Grundlage differenzierter Zeitanalyse taugten.

44 Auch die einstigen Großkausalitäten von >Gesellschaft $<$, >System<, >Fortschritt $</>$ Steigerung $<$ usw. sind allenfalls zu einer inflatorischen Rede entgrenzt.

45 Und diese sind nicht erfahrungsgehaltlich oder argumentativ aufeinander bezogen, sondern jede mit einer ganz neuen Deutung ansetzend. 